\title{
The art of responding to reviews
}

\author{
Between submission of your paper and its publication stands the peer-review process. Responding to reviewer \\ comments effectively will help to make this stage an edifying rather than painful experience.
}

A convoluted back-and-forth between authors and reviewers can easily mean months of delay, not to mention effort, anger and anxiety. As editors, we are instrumental in keeping the process on track. We check reviewers for conflicts of interest, keep in touch to ensure reports are on time, and ask them to be as objective and constructive as they can. We also carefully read and evaluate reviewer comments, and request rewrites of inappropriately worded reports. However, authors, too, can make a big difference to how their papers fare with reviewers. Like everyone involved, they have a responsibility to keep the exchanges fruitful and dispassionate, even when perspectives on the science differ.

We were thus pleased when one of our authors recently appreciated our invitation to reconsider the language of their rebuttal letter, and understood that "it would take superhuman objectivity [from the negative reviewer] to put aside being called 'obtuse' (several times)". To clarify the process: the primary purpose of the rebuttal is to convince the reviewers that all of the points raised have been addressed in an adequate manner that is, incorporated or refuted. We usually send the authors' responses to the previous round of review back to all reviewers for their consideration, so the responses should be worded for reviewer consumption. Here, we would like to share our top tips on how to write a rebuttal letter that is likely to avoid unnecessary wrinkles and detours.

Seek clarity. Read the reports and the decision letter from your editor more than once. Leave at least one good night's sleep between readings, before you even start thinking about your rebuttal. If, despite all your efforts and consultation with a friendly colleague, an important comment is not clear or too general to be addressed (such as "section X is bad"), feel free to ask the editor to seek clarification from the reviewer with a specific question.

Tone of response. Respond in language that conveys your conviction that the reviewers are knowledgeable, well-meaning people who have given up their time to assess your

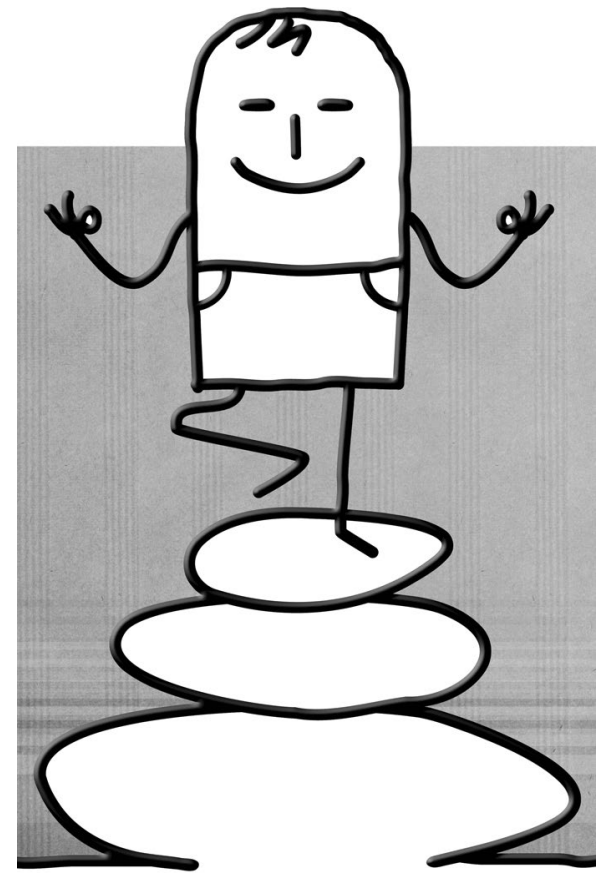

Credit: NLshop / Alamy Stock Photo

paper. If you do not hold that conviction, do not let that show in your response, but raise your concerns in a separate cover letter to the editor, with as much evidence and specifics as you can collate.

Follow through. A thorough response to the reviewers is only half the ticket. Ensure that in the manuscript itself, you have also addressed all of the comments and requests for clarification. If the reviewers struggled to follow your reasoning, it is likely that others will too.

Make it easy for editors and reviewers. The easier it is to assess the revision, the more likely you are to receive a quick answer. It helps to highlight key responses to the most crucial issues at the top; to list changes to the manuscript in your rebuttal letter with the relevant comment and response; and to include line numbers in the revised manuscript and point to them in the response where appropriate.
Stand up for your science. Reviewers are as fallible as everybody else. If you believe they made a mistake, explain your reasoning politely and provide compelling support for your point of view. If you think there has been a misunderstanding, explain. There is no point in being dismayed about errors: most likely the subject matter is complicated and you know more about the specific piece of work presented in your paper than the reviewer.

Make it complete, keep it concise. Editors and reviewers want to see that all points have been acknowledged and addressed, so do not be tempted to ignore what you do not like. On the other hand, more volume does not make your reasoning sharper, and a long-winded thirty-page rebuttal is more likely to lead reviewers down the path of procrastination than a crisp and clear twopage response. Write as much as you have to, but as little as possible.

Focus on the substance, not the personalities. Try to keep yourself from guessing who the reviewers are and what their intentions might be: you have little to win and much to lose. We find that the rate of failure with such guesses - when they are expressed to us - is high. Second-guessing identities is more likely to distract from responding effectively than to help your case.

If your experience is limited - either with writing rebuttals or with being on the other side, as a peer reviewer - we would recommend that you do not rely solely on our top tips, but in addition find a mentor or colleague to go through your proposed rebuttal and advise.

It is worth focusing your energy to make your paper as convincing as you can, and the reviewers' comments are there to help with that. Ultimately, it is the strength of your science that will determine whether your paper is published, and where.

Published online: 30 May 2019 https://doi.org/10.1038/s41561-019-0386-7 